\title{
PRÁTICAS INTEGRATIVAS E COMPLEMENTARES - ANÁLISE DOCUMENTAL E O OLHAR DE PROFISSIONAIS DA SAÚDE
}

\author{
Integrative and complementary practices - documentary analysis and health \\ professionals' look
}

\section{Prácticas integrativas y complementarias - análisis documental y la mirada de profesionales sanitarios}

\author{
Luciana Elisabete Savaris (iD \\ Universidade Federal do Paraná - UFPR - Curitiba (PR) - Brasil \\ Beatriz Böger \\ Universidade Federal do Paraná - UFPR - Curitiba (PR) - Brasil
}

Anna Cláudia Savian

Universidade Federal do Paraná - UFPR - Curitiba (PR) - Brasil

Andressa Siqueira Jansen (iD

Universidade Federal do Paraná - UFPR - Curitiba (PR) - Brasil

Milene Zanoni da Silva iD

Universidade Federal do Paraná - UFPR - Curitiba (PR) - Brasil

\section{RESUMO}

Objetivo: Investigar a implantação das práticas integrativas e complementares e avaliar o uso, formação e aceitação dessas práticas pelos profissionais que atuam na Atenção Primária à Saúde (APS). Métodos: Estudo dividido em dois momentos: a) documental retrospectivo, realizado pela leitura sistemática dos relatórios das Conferências Municipais de Saúde (2005-2015) de Curitiba, Paraná, Brasil, identificando propostas relativas à Política Nacional de Práticas Integrativas e Complementares (PNPIC); b) quantitativo transversal, realizado em um Distrito Sanitário de Saúde do município analisado, em 2017, com 240 profissionais de Unidades Básicas de Saúde (UBS). Coletaram-se os dados a partir de entrevista semiestruturada, a qual incluiu variáveis socioeconômicas e as práticas integrativas e complementares (PIC): conhecimento da PNPIC; formação em PIC; desenvolvimento de PIC na APS; acesso ao encaminhamento; ser usuário de PIC; opinião sobre benefícios das PIC na APS. Os resultados foram analisados por meio de frequências e percentuais. Resultados: Das 2.975 propostas analisadas, identificaram-se 20 referentes à PINPIC, o que representa $0,71 \%$ do total. Os dados primários apontam que $81,3 \%(n=195)$ dos profissionais desconhecem a PNPIC, 98,7\% $(n=237)$ acreditam nos benefícios da oferta da APS, 5,8\% $(n=14)$ possuem formação e, destes, 2,1\% ( $n=5)$ ofertam PIC na APS. Conclusão: A proposta de implantação de PIC no município investigado é incipiente. No distrito sanitário estudado existe pouca utilização, embora haja um crescente interesse e aceitação das PIC pelos profissionais da saúde que atuam na APS.

Descritores: Terapias Complementares; Homeopatia; Acupuntura; Sistema Único de Saúde.

\section{ABSTRACT}

Objective: To investigate the implementation of integrative and complementary practices and assess the use, training and acceptance of these practices by professionals working in Primary Health Care (PHC). Methods: The study was held in two stages: (a) a retrospective documentary analysis was done through systematic reading of the reports of the Municipal Health Conferences (2005-2015) held in Curitiba, Paraná, Brazil to identify proposals related to the National Integrative and Complementary Practices Policy (Política Nacional de Práticas Integrativas e Complementares - PNPIC); (b) a cross-sectional quantitative study was conducted in a Sanitary District located in the analyzed municipality in 2017 with 240 professionals in eight PHC centers. Data were collected through semi-structured interviews that addressed socioeconomic variables and integrative and complementary practices (ICP): knowledge of PNPIC; ICP training; ICP practice in PHC; access to referral; being a user of ICP; opinion on benefits of ICP in PHC. The results were analyzed using frequencies and percentages. Results: Of the 2,975 proposals analyzed,

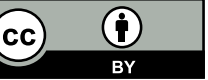


20 were related to the PINPIC, which represents $0.71 \%$ of the total. Primary data indicate that $81.3 \%$ ( $n=195)$ of professionals are unaware of the PNPIC, 98.7\% $(n=237)$ believe in the benefits of its provision in PHC, $5.8 \%(n=14)$ have been trained and $2.1 \%(n=5)$ of these offer ICP in PHC. Conclusion: The proposal for the implementation of ICP in the analyzed municipality is incipient. Although there is a growing interest and acceptance of PIC by health professionals working in PHC, it is little used in the analyzed Sanitary District.

Descriptors: Complementary Therapies; Homeopathy; Acupuncture; Unified Health System.

\section{RESUMEN}

Objetivo: Investigar la implantación de prácticas integrativas y complementarias y evaluar el uso, la formación y la aceptación de esas prácticas por profesionales de la Atención Primaria de Salud (APS). Métodos: Estudio dividido en dos momentos: a) documental retrospectivo realizado a través de la lectura sistemática de los informes de las Conferencias Municipales de Salud (2005-2015) de Curitiba, Paraná, Brasil, identificando propuestas de la Política Nacional de Prácticas Integrativas y Complementarias (PNPIC); b) cuantitativo transversal realizado en un Distrito Sanitario de Salud del municipio analizado en 2017 con 240 profesionales de Unidades Básicas de Salud (UBS). Se recogieron los datos a partir de entrevista semiestructurada en la cual se incluyeron las variables socioeconómicas y las prácticas integrativas y complementarias (PIC): conocimiento de la PNPIC; formación en PIC; desarrollo de PIC en la APS; acceso a la consulta; ser usuario de PIC; opinión sobre los beneficios de las PIC en la APS. Se analizaron los resultados por frecuencias y porcentajes. Resultados: De las 2.975 propuestas analizadas se ha identificado 20 referentes a la PINPIC lo que representa el 0,71\% del total. Los datos primarios señalan que el 81,3\% ( $n=195)$ de los profesionales desconocen la PNPIC, el 98,7\% $(n=237)$ creen en los beneficios de la oferta de la APS, el $5,8 \%$ $(n=14)$ tienen formación y, de ellos, el 2,1\% $(n=5)$ ofertan PIC en la APS. Conclusión: La propuesta de implantación de PIC en el municipio investigado es incipiente. En el distrito sanitario estudiado hay poca utilización aunque haya un creciente interés y aceptación de las PIC por los profesionales sanitarios que trabajan en la APS.

Descriptores: Terapias Complementarias; Homeopatía; Acupuntura; Sistema Único de Salud.

\section{INTRODUÇÃO}

As práticas integrativas e complementares (PIC) compreendem um conjunto heterogêneo de saberes, práticas e produtos que não pertencem ao escopo da medicina convencional ${ }^{(1)}$. Surgem no Brasil numa perspectiva de integralidade na atenção à saúde da população com foco em estratégias e formas de produzir saúde no âmbito individual e coletivo(2). A proposta de integrar as PIC nos serviços públicos de saúde aparece na Conferência Internacional de Alma-Ata (1978), de modo concomitante aos debates relacionados à desigualdade social, falta de acesso aos serviços básicos de saúde e elevada taxa de mortalidade ${ }^{(3)}$. Nesse período também surgem questionamentos acerca do modelo biomédico, e a saúde passa a ser entendida mais do que como ausência de doença, incorporando aspectos da qualidade de vida, bem-estar emocional, felicidade e vitalidade. Aspectos subjetivos do adoecer e diferentes dimensões do sujeito passam a ser valorizados ${ }^{(4,5)}$.

No ano de 1986, na I Conferência Internacional da Promoção da Saúde em Ottawa, o modelo assistencial e a concepção da saúde-doença como processo são debatidos, bem como a proposta de articulação entre os saberes técnicos e populares para o alcance da integralidade do cuidado. Logo, as PIC surgem como recursos, técnicas e sistemas terapêuticos que destacam outras dimensões do sujeito e incorporam diferentes racionalidades médicas ${ }^{(6)}$.

No Brasil, encontram-se registros de debates sobre a inserção das PIC no Sistema Único de Saúde (SUS) desde a década de 1980, ganhando força nas deliberações da $8^{\circ}$ Conferência Nacional de Saúde (1986), por meio de relatórios e portarias. Em fevereiro de 2006, foi aprovado pelo Conselho Nacional de Saúde o documento que embasou a criação da Política Nacional de Práticas Integrativas e Complementares (PNPIC) no SUS, publicado em forma de Portaria Ministerial $n^{\circ}$ 971, em 03 de maio de 2006, e $n^{\circ} 1.600$, de 17 de julho de 2006 ${ }^{(7)}$.

Após a aprovação da PNPIC, a efetivação das ofertas de PIC no SUS vem ocorrendo de forma gradativa. No ano de 2017, o Ministério da Saúde relata 8.200 estabelecimentos de saúde, distribuídos em $54,2 \%$ municípios brasileiros (3.018), ofertando PIC. Atualmente, as PIC estão presentes em $100 \%$ das capitais por iniciativa das gestões locais. O Brasil tem 29 PIC habilitadas em sua política nacional, despontando como um dos países com mais práticas aprovadas ${ }^{(8)}$. Alguns aspectos, como o baixo incentivo financeiro, o pouco investimento em formação, a baixa avaliação e monitoramento, em especial referente à inserção na Atenção Primária à Saúde (APS), têm sido questionados na PNPIC ${ }^{(1)}$.

A PNPIC e a Política de Promoção da Saúde se articulam, visto que ambas são campos de propostas, ideias e práticas que partem de uma concepção ampliada do processo saúde-doença e seus determinantes, especialmente 
do autocuidado ${ }^{(9)}$. Ambas articulam saberem técnicos e populares, considerando o contexto sóciohistórico, em busca de produzir melhorias na qualidade de vida das pessoas ${ }^{(10,11)}$. As PIC são um valioso recurso para a promoção da saúde ${ }^{(9)}$.

Apesar do desafio da sua incorporação no SUS, a busca e a utilização das PIC são crescentes entre as populações e profissionais da saúde, não apenas no Brasil, mas em todo o mundo ${ }^{(12)}$. Contudo, atualmente tem existido intenso debate acerca da manutenção ou não das PIC no SUS, apesar da Organização Mundial da Saúde (OMS) recomendar e valorizar a implementação das medicinas tradicionais complementares (MTC) em seus Estadosmembros, bem como indicar a necessidade de criação de uma base de conhecimento para uma gestão ativa das $\mathrm{PIC}^{(13)}$. Logo, este artigo objetivou investigar a implantação das práticas integrativas e complementares e avaliar o uso, formação e aceitação dessas práticas pelos profissionais que atuam na Atenção Primária à Saúde.

\section{MÉTODOS}

Trata-se de uma pesquisa quantitativa de dados, dividida em duas etapas: a primeira etapa, documental e retrospectiva; a segunda, de natureza quantitativa, com delineamento transversal.

A etapa documental aconteceu no primeiro semestre de 2017 e abrangeu a coleta de dados secundários a partir da seleção, organização, leitura sistemática e análise dos relatórios finais das Conferências Municipais de Saúde de Curitiba, sendo a $8^{a}$ realizada em 2005 e a $13^{a}$ em 2015, totalizando seis documentos públicos analisados no presente estudo, todos disponibilizados no site do Conselho Municipal de Saúde de Curitiba ${ }^{(14)}$.

A decisão pelo período de 2005-2015 deu-se em função do ano de aprovação da PNPIC (2006) até o último relatório vigente (2015), com o intuito de se analisar o impacto da PNPIC em âmbito municipal. Nesses documentos, houve busca ativa por termos/palavras-chave relacionados às PIC para identificação das propostas. As palavraschaves utilizadas foram: "tradicional", "integrativo", "popular", "natural", "não hegemônico", "não convencional", "alternativo", "complementar" - além dos termos "homeopatia", "fitoterapia", "acupuntura", "termalismo", "crenoterapia" e "medicina antroposófica". Foram excluídas as citações fora do contexto das propostas/deliberações sobre PIC.

A partir dos nós críticos para a implementação da PNPIC, apontados por autores que publicam na área ${ }^{(4,15,16)}$, realizou-se a seleção de três categorias a priori, a citar: destinação de recursos financeiros, estudos/pesquisas e formação/qualificação profissional, as quais seriam importantes para avaliar em que medida esses pontos críticos estão sendo contemplados nas propostas analisadas.

Na sequência ao estudo documental, realizou-se a pesquisa de campo, de delineamento transversal e observacional, no lócus prioritário de execução da PNPIC, ou seja, em Unidades de Saúde da Família (USF). A população de estudo constituiu-se pelo total de profissionais do Distrito Sanitário Tatuquara (DS Tatuquara) (n=253), que compõem as Equipes Saúde da Família de oito unidades de Saúde da Família. As equipes incluem: agentes comunitários de saúde $(n=64)$, auxiliares de Enfermagem $(n=77)$, enfermeiros $(n=24)$, autoridades sanitárias locais $(n=6)$, técnicos de Enfermagem ( $n=77)$, enfermeiros $(n=24)$, médicos $(n=22)$, técnicos e auxiliares de saúde bucal $(n=31)$ e cirurgiõesdentistas $(n=16)$. O DS Tatuquara é composto por três bairros, totalizando 82 mil habitantes, o que corresponde a 4,7\% da população total de Curitiba, que, segundo dados do IBGE (2010), são de 1.751.907 habitantes.

A seleção do território baseou-se no DS Tatuquara contar com $100 \%$ das suas UBS com ESF e ter apresentado o maior índice de vulnerabilidade (IVAB) do município, que leva em conta as variáveis sobre acesso ao trabalho, renda e escolaridade. Além disso, o DS Tatuquara é o mais afastado geograficamente do centro da cidade, o que sugere a necessidade de oferta diversificada de cuidados no território para viabilizar o acesso à saúde aos seus usuários.

Os critérios de inclusão da pesquisa eram: (a) ter idade mínima de 18 anos; (b) ser funcionário efetivo da Secretaria Municipal de Saúde de Curitiba, (c) estar em atividade funcional nas USF do distrito do Tatuquara e (d) aceitar participar da pesquisa. Por sua vez, os critérios de exclusão eram: possuir outro vínculo com a Unidade Básica de Saúde, como de residência, estágio ou similar; ser afastado por algum tipo de licença (férias no período da pesquisa, licença de saúde ou licençaprêmio); e desistir da pesquisa durante a entrevista.

A equipe, treinada e capacitada, com supervisão da pesquisadora principal, realizou a coleta dos dados por meio de entrevistas semiestruturadas, realizadas entre os meses de junho e setembro de 2017. Além disso, realizou-se pré-teste para ajustes no conteúdo e na ordem das perguntas.

As questões analisadas no estudo tratavam das variáveis sociodemográficas (idade, sexo, estado civil, filhos, escolaridade, tempo de atuação, cargo e horas de trabalho) e da temática das PIC, a citar: (1) Você conhece a PNPIC? (2) Possui alguma formação em PIC? Se sim, qual? (3) Você desenvolve algumas PIC na sua prática profissional no SUS? Se sim, quais? (4) Você tem acesso ao encaminhamento do seu paciente para algumas PIC? 
Se sim, quais? (5) Você já foi usuário de algumas PIC? (6) Em sua opinião, as PIC podem trazer benefícios aos usuários se implementadas na APS? Se sim, quais?

Para efeitos de análise criou-se a categoria "envolvimento com PIC", que se refere aos profissionais que possuem formação e/ou já foram usuários de alguma prática. Foi analisada a temática PIC a partir das categorias "médico", "ACS" e "outros profissionais", tendo como hipótese maior frequência de formação e prática na APS da categoria médica, por conta da acupuntura e homeopatia serem especialidades médicas.

Realizou-se a análise quantitativa dos dados por meio da epidemiologia descritiva. Os resultados foram descritos por média, desvio padrão, mediana e amplitude, e as variáveis categóricas, por frequência e percentual. A avaliação da associação entre variáveis categóricas considerou o teste de qui-quadrado ou teste exato de Fisher $(\mathrm{p}<0,05)$. Os dados foram analisados com o programa Stata/SE, versão 14.1 (Stata Corp LP, USA).

Esta pesquisa obteve a aprovação do Comitê de Ética da Universidade Federal do Paraná (UFPR), Parecer $\mathrm{n}$. 2.031.061, de abril de 2017, e do Comitê de Ética da Secretaria Municipal de Saúde (SMS), Parecer n. ${ }^{\circ} 2.086 .711$, de maio de 2017.

\section{RESULTADOS}

Os achados da investigação documental de dez anos da Conferência Municipal de Saúde (CMS) de Curitiba evidenciaram 2.975 propostas aprovadas no município de Curitiba, sendo que deliberações com PIC foram quase inexistentes nesse período, totalizando $0,71 \%(n=20)$. O ano de 2013 teve a maior prevalência, de $2,4 \%(n=2)$, e 2007 teve a menor, de $0,24 \%(n=0,2)$.

Os termos mais utilizados para se referir às PIC foram: práticas complementares (2); medicinas naturais (2); práticas integrativas e complementares (2); terapias alternativas (2); práticas integrativas e alternativas (1) e terapias integrativas, alternativas e complementares (1). As PIC mais citadas foram: fitoterapia (4); homeopatia (3); acupuntura (3) e plantas medicinais (1).

Dentre as deliberações, quando categorizadas a partir dos nós críticos da implementação das PIC, apenas duas tratam da destinação de recursos, sendo uma de estudo/pesquisa e uma da formação dos profissionais, dados ínfimos.

No Quadro I está descrito o quantitativo de propostas aprovadas na CMS entre 2005-2015, percentual relativo a deliberações que envolvem PIC e seu detalhamento.

No que se refere à coleta de dados primários da segunda etapa da pesquisa, dos 253 profissionais de saúde lotados em ESF no território estudado, foram entrevistados 240 , o que corresponde a $94,9 \%$ dos trabalhadores. Dos 13 que não participaram da entrevista, houve quatro perdas por recusa, sendo três de médicos e uma de enfermeiro, além de nove exclusões em função de afastamento por licença de saúde e licença-prêmio.

$\mathrm{Na}$ Tabela I, quanto ao perfil demográfico e de atuação profissional, verificou-se um predomínio do sexo feminino (88,3\%; $n=212)$, de pessoas com companheiro (a) $(65,8 \% ; n=158)$ e filhos $(83,8 \% ; n=201)$. Quanto à escolaridade, $51,3 \%(n=123)$ dos profissionais apresentaram nível médio, seguidos por $48,8 \%$ com nível superior.

Na Tabela II, os dados apresentados mostram que $53,8 \%(n=129)$ dos profissionais pesquisados apresentaram envolvimento com PIC, 81,3\% $(n=195)$ desconhecem a PNPIC e a quase totalidade dos entrevistados $(98,7 \%, n$ $=152$ ) acredita que a implantação de tais práticas no seu território traria benefícios aos usuários. Apesar disso, a formação é baixa $(5,8 \%, n=14)$ e apenas $2,1 \%(n=5)$ utilizam as PIC em seu espaço de trabalho.

Ao se estratificar por categoria profissional, os médicos, assim como os demais profissionais, têm envolvimento duas vezes maior com PIC em comparação aos agentes comunitários $(p<0,0001)$. No mesmo sentido, as variáveis conhecer a PNPIC, possuir formação, encaminhar a PIC e ser usuário foram estatisticamente maiores entre os médicos em comparação aos agentes comunitários de saúde. Vale destacar que, apesar de todos $(100 \% ; n=64)$ os agentes comunitários de saúde avaliarem que as PIC podem trazer benefícios para os usuários, apenas $29,7 \%$ $(\mathrm{n}=19)$ utilizam as práticas.

Na Tabela III, que detalha a formação em PIC, verificou-se que $28,6 \%$ deles $(n=4)$ são homeopatas. Quanto à possibilidade de encaminhamento, $63,4 \%(n=41)$ dos entrevistados afirmaram ser possível, sendo que, destes, $63,4 \%(n=26)$ relatam que o tratamento disponível é a acupuntura.

$\mathrm{Na}$ Tabela IV encontra-se a descrição do que foi citado como benefícios das PIC. Observa-se que 22,1\% ( $n=$ 53) dos profissionais acreditam que elas possam melhorar as condições de saúde mental, seguidos por 20,8\% ( $n$ $=50$ ) que refere haver diminuição no uso de medicação. Quanto à qual PIC deveria ser implementada no território, $14,6 \%(n=35)$ apontam a acupuntura. 
Quadro I - Perfil descritivo das propostas aprovadas envolvendo práticas integrativas e complementares em saúde nas Conferências Municipais de Saúde de Curitiba (2005-2015). Curitiba, 2017.

\begin{tabular}{|c|c|c|c|}
\hline Ano & $\begin{array}{c}\text { Propostas } \\
\text { aprovadas (n) }\end{array}$ & $\begin{array}{l}\text { Total de propostas } \\
\text { aprovadas referentes } \\
\text { às PIC } n(\%)\end{array}$ & Propostas aprovadas que envolvem PIC \\
\hline 2005 & 596 & $03(0,5)$ & $\begin{array}{l}\text { 162. Enviar a tese }\{\ldots . .\} \text { da Associação de Farmacêuticos } \\
\text { Homeopatas do PR para CES } \\
\text { 289. \{...\} Garantir a continuação e a implementação } \\
\text { do programa de fitoterapia da SMS }\{\ldots\} \\
\text { 337. Estimular a incorporação de práticas saudáveis } \\
\text { e acesso às medicinas naturais e práticas } \\
\text { complementares }\{\ldots\}\end{array}$ \\
\hline 2007 & 829 & $02(0,24)$ & $\begin{array}{l}\text { 339. }\{\ldots\} \text { Garantir a continuação e a implementação } \\
\text { do programa de fitoterapia da SMS }\{\ldots\} \\
438 \text {. Estimular a incorporação de práticas saudáveis } \\
\text { e acesso às medicinas naturais e práticas } \\
\text { complementares }\{\ldots\}\end{array}$ \\
\hline 2009 & 390 & $01(0,25)$ & 279. Implantar a PNPIC no SUS Curitiba \\
\hline 2011 & 403 & $02(0,49)$ & $\begin{array}{l}\text { 07. }\{\ldots\} \text { incluir a acupuntura em Odontologia } \\
220 \text {. Sensibilizar os homens para atividades de } \\
\text { promoção }\{\ldots\} \text { incluindo terapias alternativas e terapias } \\
\text { corporais }\end{array}$ \\
\hline 2013 & 331 & $08(2,40)$ & $\begin{array}{l}\text { 33. }\{\ldots . .\} \text { promoção e prevenção, contemplando PICS } \\
\text { para todos os segmentos }\{\ldots\} \\
\text { 103. }\{\ldots\} \text { Incluir a acupuntura em Odontologia }\{\ldots\} \\
\text { 142. Criação centro para PCd com terapias alternativas } \\
\{\ldots\} \\
\text { 143. }\{\ldots\} \text { Incluir a acupuntura em Odontologia }\{\ldots\} \\
\text { 209. }\{\ldots\}\} \text { Incorporar medicamentos fitoterápicos e } \\
\text { homeopáticos } \\
\text { 210. Implantar a PNPIC em todos os níveis de atenção } \\
\{\ldots\} \\
\text { 211. Priorizar profissionais com titulação para atuar com } \\
\text { PICS }\{\ldots\} \text { qualificando os demais }\{\ldots\} \text { para viabilizar a } \\
\text { implantação destes serviços } \\
\text { 212. Ampliar a inserção de códigos existentes SAI/SUS } \\
\text { para registro de PICS }\{\ldots\}\end{array}$ \\
\hline 2015 & 246 & $04(1,62)$ & $\begin{array}{l}\text { 59. }\{\ldots\} \text { Incluir a acupuntura em Odontologia }\{\ldots\} \\
\text { 107. }\{\ldots\} \text { implantar as terapias integrativas e } \\
\text { complementares com apoio irrestrito da SMS } \\
\text { 131. ASMS deverá realizar um estudo para a implantação } \\
\text { de plantas medicinais e fitoterápicos nas US }\{\ldots\} \\
\text { 132. Incluir acupuntura e outras PICS na carteira de } \\
\text { serviços da APS }\{\ldots\}\end{array}$ \\
\hline Total & 2.795 & $20(0,71)$ & \\
\hline
\end{tabular}


Tabela I - Variáveis demográficas e de atuação profissional dos profissionais da saúde da Equipe de Saúde da Família. Curitiba, 2017.

\begin{tabular}{|c|c|c|c|}
\hline Variável & Classificação & $\mathbf{n}$ & $\%$ \\
\hline Idade (anos) & Média $\pm \mathrm{dp}(\min -\max )$ & $36,1 \pm 9,7$ & $(23,8-69,1)$ \\
\hline \multirow[t]{2}{*}{ Sexo } & Masculino & 28 & 11,7 \\
\hline & Feminino & 212 & 88,3 \\
\hline \multirow[t]{2}{*}{ Estado civil } & Sem companheiro(a) & 82 & 34,2 \\
\hline & Com companheiro(a) & 158 & 65,8 \\
\hline \multirow[t]{2}{*}{ Filhos } & Sim & 201 & 83,8 \\
\hline & Não & 39 & 16,3 \\
\hline \multirow[t]{2}{*}{ Escolaridade } & Médio incompleto/completo & 123 & 51,3 \\
\hline & Superior incomp/completo/pós-grad & 117 & 48,7 \\
\hline Tempo da atuação (anos) & Média $\pm \mathrm{dp}(\min -\max )$ & $16,4 \pm 8,8$ & $(0,3-41)$ \\
\hline \multirow[t]{7}{*}{ Cargo } & Auxiliar de Enfermagem & 77 & 32,1 \\
\hline & ACS & 64 & 26,7 \\
\hline & Enfermeiro & 24 & 10,0 \\
\hline & Médico & 22 & 9,2 \\
\hline & ASB/TSB & 31 & 12,9 \\
\hline & Cirurgião-dentista & 16 & 6,7 \\
\hline & ASL & 6 & 2,5 \\
\hline \multirow[t]{2}{*}{ Horas de trabalho } & De 30 a 40 & 233 & 97,1 \\
\hline & Mais de 40 & 7 & 2,9 \\
\hline
\end{tabular}

Dp: desvio-padrão; min: mínimo; max; máximo; incomp: incompleto; pós-grad: pós-graduação; ACS: agentes comunitários de saúde; ASB: auxiliar de saúde bucal; TSB: técnico de saúde bucal; ASL: autoridades sanitárias locais 
Tabela II - Comparação entre agentes comunitários, médicos e outros profissionais quanto a variáveis relacionadas às práticas integrativas e complementares. Curitiba, 2017.

\begin{tabular}{|c|c|c|c|c|c|}
\hline Variável & $\begin{array}{c}\text { Total } \\
(n=240)\end{array}$ & $\begin{array}{c}\text { Agente } \\
\text { comunitário } \\
\text { de saúde } \\
(n=64)\end{array}$ & $\begin{array}{c}\text { Médicos } \\
(n=22)\end{array}$ & $\begin{array}{c}\text { Outros } \\
\text { profissionais } \\
(n=154)\end{array}$ & Valor de $\mathrm{p}^{*}$ \\
\hline
\end{tabular}

\section{Envolvimento com PIC}

Sim

Não

\section{Conhece a PNPIC}

$\operatorname{Sim}$

Não

\section{Possui formação em PIC}

Sim

Não

\section{Desenvolve PIC}

Sim

Não

\section{Acesso a encaminhamento para PIC}

Sim

Não

\section{Usuário de PIC}

Sim

Não

\section{Benefícios da PIC na APS}

$\begin{array}{ccccc}\text { Sim } & 237(98,7) & 64(100) & 21(95,5) & 152(98,7) \\ \text { Não } & 03(1,3) & 0(0) & 1(4,6) & 2(1,3)\end{array}$

*:Teste de qui-quadrado, $p<0,05 ;(-)$ : Teste não aplicável; PIC: práticas integrativas e complementares; APS: Atenção Primária à Saúde; PNPIC: Política Nacional de Práticas Integrativas e Complementares 
Tabela III - Perfil de formação, realização e encaminhamento para práticas integrativas e complementares na Equipe de Saúde da Família entre profissionais de Curitiba. Curitiba, 2017.

\begin{tabular}{|c|c|c|c|c|}
\hline Variáveis & TOTAL & $\begin{array}{c}\text { Agentes } \\
\text { comunitários de } \\
\text { Saúde }(n=1)\end{array}$ & $\begin{array}{l}\text { Médicos } \\
\qquad(n=4)\end{array}$ & $\begin{array}{l}\text { Outros } \\
\text { profissionais }(n=9)\end{array}$ \\
\hline \multicolumn{5}{|l|}{ Formação em PIC (n=14) } \\
\hline Homeopatia & $4(28,6)$ & - & $3(75)$ & $1(11,1)$ \\
\hline Acupuntura & $3(21,4)$ & - & $1(25)$ & $2(22,2)$ \\
\hline Massoterapia & $3(21,4)$ & $1(100)$ & - & $2(22,2)$ \\
\hline Reiki & $2(14,3)$ & - & - & $2(22,2)$ \\
\hline Shiatzu & $1(7,1)$ & - & - & $1(11,1)$ \\
\hline Desenvolvimento em PIC (n=5) & & $\begin{array}{l}\text { Agentes } \\
\text { comunitários }(n=0)\end{array}$ & $\begin{array}{l}\text { Médicos } \\
\qquad(n=2)\end{array}$ & $\begin{array}{c}\text { Outros } \\
\text { profissionais } \\
(n=3)\end{array}$ \\
\hline Homeopatia & 2 & - & $2(100)$ & \\
\hline Auriculoterapia & 1 & - & - & $1(33,3)$ \\
\hline Acupuntura & 1 & - & - & $1(33,3)$ \\
\hline $\begin{array}{l}\text { Acesso a encaminhamento para } \\
\text { PIC }(n=41)\end{array}$ & & $\begin{array}{l}\text { Agentes } \\
\text { comunitários de } \\
\text { Saúde }(n=4)\end{array}$ & $\begin{array}{l}\text { Médicos } \\
\qquad(n=9)\end{array}$ & $\begin{array}{l}\text { Outros } \\
\text { profissionais }(n=27)\end{array}$ \\
\hline Acupuntura & $26(63,4)$ & $2(50,0)$ & $4(44,4)$ & $20(74,1)$ \\
\hline Homeopatia e acupuntura & $9(21,9)$ & $1(25,0)$ & $3(33,3)$ & $5(18,5)$ \\
\hline Homeopatia & $4(9,8)$ & $1(25,0)$ & $1(11,1)$ & $2(7,4)$ \\
\hline Fitoterapia & $1(2,4)$ & - & $1(11,1)$ & - \\
\hline Não citou & $1(2,4)$ & - & - & - \\
\hline
\end{tabular}

PIC: práticas integrativas e complementares

Tabela IV - Variáveis relativas aos benefícios das práticas integrativas e complementares em saúde segundo os profissionais da Equipe de Saúde da Família. Curitiba, 2017.

\begin{tabular}{lccc}
\hline Variáveis & Classificação & $\mathbf{n}$ & \% \\
\hline Benefícios (n=240) & & & \\
Melhora das condições de saúde mental & Sim & 53 & 22,1 \\
Diminuição do uso de medicação & Sim & 50 & 20,8 \\
Manejo da dor aguda e crônica & Sim & 47 & 19,6 \\
Melhora da qualidade de vida e bem-estar & Sim & 31 & 12,9 \\
Redução do estresse & Sim & 22 & 9,2 \\
Qual PIC traria mais benefícios (n=240) & & & \\
Acupuntura & Sim & 35 & 14,6 \\
Yoga & Sim & 6 & 2,5 \\
Homeopatia & Sim & 9 & 3,8 \\
Florais & Sim & 4 & 1,7 \\
Reiki & Sim & 1 & 0,4 \\
\hline
\end{tabular}

PIC: práticas integrativas e complementares

\section{DISCUSSÃO}

Após análise dos Relatórios Finais da CMS de 2005-2015 no presente estudo, verificou-se que as propostas com PIC foram quase inexistentes $(0,71 \%)$ e que, destas, poucas abordavam os nós críticos para a implantação efetiva da PNPIC, como financiamento e formação/qualificação dos profissionais. As deliberações foram pouco propositivas e se repetiram sistematicamente ao longo das conferências, o que evidência periferização da temática frente às políticas e ao Plano Municipal de Saúde. 
Cabe ressaltar que tanto a política quanto o Plano Municipal de Saúde são embasados nas propostas aprovadas na CMS, incluindo a destinação dos recursos financeiros, essenciais para efetivação das políticas, ficando evidente que a PNPIC não foi tomada como prioritária pelos gestores públicos ${ }^{(17,18)}$.

Sendo assim, os resultados da atual pesquisa sugerem que os diferentes segmentos (gestores, trabalhadores e usuários) ou desconhecem a PNPIC, ou estão pouco apropriados/engajados na discussão da inserção das PIC no Brasil, apesar de desde a $8^{\text {a }}$ Conferência Nacional de Saúde já se defender a necessidade de ofertar outras práticas e estratégias de cuidado, e a Organização Mundial de Saúde (OMS), desde a Conferência de Alma Ata, na década de 70, valorizar e recomendar aos Estadosmembros a implantação das medicinas tradicionais, complementares e alternativas nos sistemas nacionais de saúde ${ }^{(3)}$.

Os relatórios finais da CMS do município de Curitiba confirmam que existe um grande desafio na institucionalização dessas práticas, ainda que o Brasil tenha recebido lugar de destaque entre os países com sistemas universais de saúde com a elaboração da PNPIC ${ }^{(1)}$. Portanto, transpor a Política Nacional em ações efetivas no âmbito municipal ainda é um desafio a ser superado, com o necessário fortalecimento da atenção básica.

Com base no objetivo estratégico da OMS 2014-2023(3), que trata da necessidade de criar uma base de conhecimentos para a gestão ativa de PIC através de políticas públicas adequadas, realizou-se a segunda etapa da pesquisa, através da coleta de dados primários para investigar o conhecimento, a aceitação e a utilização das PIC por profissionais que atuam na APS.

Os profissionais pesquisados no presente estudo foram predominantemente do sexo feminino, com companheiro(a), possuem filhos, trabalham, em média, 40 horas semanais, e têm mais de oito anos de atuação na saúde pública. Dividem-se entre os níveis médio e superior, apresentando escolaridade compatíveis com as funções desempenhadas, perfil semelhante ao descrito em outra pesquisa sobre PIC na região de São Paulo(18).

Referente ao conhecimento da PNPIC no estudo em questão, apenas 18,8\% afirmam conhecê-la, dado similar ao encontrado em outra pesquisa, em que $10,8 \%$ dos médicos e dos enfermeiros a conheciam ${ }^{(19)}$. Já entre os gestores, $26 \%$ conhecem a PNPIC ${ }^{(20,21)}$. As diferentes pesquisas revelam que o conhecimento da política é pequeno, sugerindo refletir se os meios adotados para sua disseminação são efetivos.

Quanto à formação dos profissionais para realizar PIC, somente 5,8\% dos entrevistados do atual estudo possuem alguma qualificação. Destes, 18,2\% são médicos e têm formação na área: homeopatas, acupunturistas e massoterapeutas. Em Campinas, Florianópolis e Recife, 83\% dos profissionais com formação em PIC são da categoria médica, também com formação em acupuntura e homeopatia, revelando a prevalência da categoria médica com formação(22).

As PIC não deveriam ser uma especialidade profissional, nem tampouco estar centrada em uma única categoria, mas uma ferramenta para o profissional, que irá eleger dentre os recursos terapêuticos os mais adequados para cada paciente dentro da APS ${ }^{(23)}$. O acesso à formação dos profissionais deveria ser viabilizado através de uma política municipal, com possibilidade para todas as categorias da equipe multiprofissional, dado não confirmado pelos achados, que demonstram a qualificação proveniente de iniciativas individuais dos profissionais de saúde, e não de oferta institucionais.

Quanto à utilização de PIC pelos profissionais que possuem formação, na sua prática no SUS, 35,7\% deles as utilizam, sendo a homeopatia e acupuntura as PIC mais utilizadas no território pesquisado. Outras pesquisas apontam a prevalência das mesmas práticas na APS. A homeopatia e a acupuntura são as práticas mais presentes na APS ${ }^{(24)}$. A acupuntura, fitoterapia e homeopatia são as práticas que os profissionais de saúde mais conhecem e têm maior experiência ${ }^{(9)}$.

Quanto ao acesso ao encaminhamento para PIC no SUS Curitiba, apenas $17,1 \%$ dos entrevistados sabiam dessa possibilidade na pesquisa em tela, o que demonstra desconhecimento da rede de saúde, seus equipamentos/ serviços ofertados, considerando que a capital paranaense possui, na atenção secundária, ambulatorial, a oferta de homeopatia e acupuntura. O ambulatório atende a todos os DS a partir de encaminhamento médico das UBS, sujeito a regulação de vagas.

Embora o município possua um ambulatório especializado, existem várias barreiras de acesso (distância, disponibilidade de vagas, entre outras), reforçando a pertinência das PIC serem uma oferta territorializada, oferecida pelos profissionais da APS. Ainda, acerca da criação de ambulatórios especializados em PIC, trata-se de uma estratégia que dificulta o acesso e a difusão dessas práticas, além de necessitar de maior número de profissionais e reduzir o potencial integrador de cuidado com a APS ${ }^{(1)}$.

Outro destaque é o uso de PIC por parte dos profissionais da saúde. Mais da metade dos entrevistados $(53,8 \%)$ da presente pesquisa relataram serem usuários de PIC, sendo que os profissionais médicos e os de outras categorias, que não ACS, são os que revelam maior uso, com $63,6 \%$ e $62,3 \%$ respectivamente, contrapondo os dados de menor uso pelos ACS $(29,7 \%)$. Outros estudos confirmam esses resultados, demonstrando a aceitação e valorização das 
PIC pelas diferentes categorias profissionais e a dificuldade de acesso para os ACS, com a escassez de ofertas disponíveis no SUS $(24,25,26)$.

Recentemente, um artigo publicado sobre a prevalência de uso de PIC por 145.580 brasileiros com 18 anos ou mais evidenciou frequência de $4,1 \%$, sendo que as principais terapias citadas foram: plantas medicinais e fitoterapia $(2,5 \%)$, acupuntura $(0,9 \%)$ e homeopatia $(0,6 \%)$. Neste estudo, os achados mostram uma prevalência de $53,8 \%$ de uso de PIC entre profissionais da saúde, uma frequência muito maior do que na população, possivelmente em função do acesso(26).

Quanto aos benefícios das PIC, quase a totalidade dos entrevistados acreditam que tais terapias são positivas para a saúde da população (98,7\%). Entre os profissionais de saúde, 92,4\% consideram as PIC eficientes. Perguntados sobre quais benefícios as PIC trariam aos usuários, citaram: a melhora nas condições de saúde mental (22,1\%), a diminuição do uso de medicação $(20,8 \%)$ e o manejo da dor crônica $(19,6 \%)^{(9)}$.

Esses dados demonstram sintonia com as evidências, pois os pacientes, em sua maioria, procuram a acupuntura por serem portadores de dores crônicas ${ }^{(12)}$. Os principais motivos que os levam a buscar PIC são: dores articulares, problemas de memória, tristeza, solidão, insônia, ansiedade e depressão. Há outros motivos que levam as pessoas a procurarem as PIC, tais como: a insatisfação com a medicina convencional; os efeitos colaterais das drogas clássicas; a busca de incremento da relação médico-paciente e a busca por um tratamento que considere a pessoa como um ser integral ${ }^{(27-30)}$.

Assim, esta pesquisa suscita a discussão das limitações do modelo biomédico vigente, baseado na doença e na prescrição, apontando para a necessidade do olhar para promoção da saúde, do bem-estar e do envolvimento do paciente no processo de cuidado. Portanto, os resultados encontrados nesta investigação vão ao encontro dos objetivos, diretrizes e prioridades de implementação da Política Nacional de Promoção da Saúde, que, em consonância com a PNPIC, destaca a necessidade de promoção da qualidade de vida e saúde em seu conceito ampliado e o estímulo de alternativas inovadoras e socialmente contributivas, com ênfase na atenção primária.

Quando questionados sobre quais PIC deveriam estar disponíveis em seu território, as mais citadas pelos profissionais pesquisados foram: a acupuntura $(14,6 \%)$, homeopatia $(3,8 \%)$, yoga $(2,5 \%)$, florais $(1,7 \%)$ e reiki $(0,4 \%)$, e todas elas estão presentes na PNPIC ${ }^{(7)}$. Essas mesmas práticas também são as mais conhecidas pelos usuários que procuram as PIC, assim como são as divulgadas pela mídia e que se enquadram na categoria práticas médicas complementares, a saber: fitoterapia, acupuntura e homeopatia ${ }^{(9)}$.

As limitações encontradas no presente estudo se deram pelo foco nos profissionais da saúde, sugerindo-se estudos complementares que abordem a percepção dos pacientes. Houve todo um cuidado para assegurar a confiabilidade na coleta dos dados e nos aspectos éticos de pesquisa com os entrevistados.

\section{CONCLUSÃO}

É possível assegurar que existe um crescente interesse e aceitação das práticas integrativas e complementares pelos profissionais da saúde que atuam na Atenção Primária à Saúde do município analisado. As práticas integrativas e complementares colaboram para efetivar a clínica ampliada e são recursos terapêuticos potentes na promoção da saúde, trazendo inúmeros benefícios na qualidade de vida, dentre eles, na área da saúde mental, o manejo da dor crônica e a redução do uso de medicação, a partir da perspectiva dos entrevistados.

Contudo, a efetivação/operacionalização da Política Nacional de Práticas Integrativas e Complementares na esfera municipal ainda encontra muitos desafios. Para ser concretizada uma política municipal, torna-se necessário o fomento e a discussão em diferentes fóruns e arenas políticas, com envolvimento de todos os atores: usuários, trabalhadores e gestores, além de haver a necessidade de se investir em pesquisas e na publicização dos resultados.

\section{AGRADECIMENTOS E CONFLITOS DE INTERESSE}

Autores agradecem à Universidade Federal do Paraná e à Secretaria Municipal de Saúde de Curitiba, que viabilizaram a pesquisa.

Não há relações financeiras ou outras que possam levar a um conflito de interesses.

\section{COLABORAÇÕES}

Luciana Elisabete Savaris e Milene Zanoni da Silva contribuíram com a elaboração do projeto, com a aquisição, análise e interpretação dos dados e a revisão crítica do manuscrito. Beatriz Böger contribuiu com a análise e 
interpretação dos dados e a redação e revisão crítica do manuscrito. Anna Cláudia Savian e Andressa Siqueira Jansen contribuíram com a aquisição, análise e interpretação dos dados e a elaboração do manuscrito.

\section{REFERENNCIAS}

1. Sousa IMC, Tesser CD. Medicina tradicional e complementar no Brasil: inserção no Sistema Único de Saúde e integração com a atenção primária. Cad Saúde Pública. 2017;33(1):1-15.

2. Tesser CD, Sousa IMC, Nascimento MC. Práticas integrativas e complementares na Atenção Primária à Saúde brasileira. Saúde Debate. 2018;42(1):174-88.

3. Organização Mundial da Saúde. Estrategia de la OMS sobre medicina tradicional 2014-2023 [Internet]. Genebra: OMS; 2013 [acesso em 2017 Jul 17]. Disponível em: http://apps.who.int/medicinedocs/documents/ s21201es/s21201es.pdf

4. Savaris LE, Martins SAK, Szpak R, Cordeiro AM, Boger B, Zanoni MS. Práticas integrativas e complementares: Incorporação no SUS e impactos na qualidade de vida da população. In: Moreira RM, Sousa LMM, Valentim OS, José HMG. Qualidade de vida e saúde em uma perspectiva interdisciplinar. Curitiba: CRV; 2019. p. 147-58.

5. Luz MT. As novas formas da saúde: práticas, representações e valores culturais na sociedade contemporânea. Rev Bras Saúde Família. 2008;9:8-19.

6. Ivo AMS, Malta DC, Freitas MIF. Modos de pensar dos profissionais do Programa Academia da Saúde sobre saúde e doença e suas implicações nas ações de promoção de saúde. Physis. 2019;29(1):e290110.

7. Brasil. Ministério da Saúde. Portaria $n^{\circ} 971$, de 4 de maio de 2006. Dispõe sobre a Política Nacional de Práticas Integrativas e Complementares (PNPIC) no Sistema Único de Saúde. Diário Oficial da União 4 de maio 2006.

8. Ministério da Saúde (BR). Mais 10 práticas integrativas são inseridas na PNPIC [Internet]. Brasília: Ministério da Saúde; 2018 [acesso em 2017 Jul 20]. Disponível em: http://www.saude.gov.br/noticias/agenciasaude/42737-ministerio-da-saude-inclui-10-novas-praticas-integrativas-no-sus

9. Lima CA, Santos AMVS, Messias RB, Costa FM, Barbosa DA, Oliveira e Silva CS, et al. Práticas integrativas e complementares: utilização por agentes comunitários de saúde no autocuidado. Rev Bras Enferm. 2018;71(6): 2682-88.

10. Amado DM, Rocha PRS, Ugarte AO, Ferraz CC, Lima MC, Carvalho FFB. Política Nacional de Práticas Integrativas e Complementares no Sistema Único de Saúde 10 anos: avanços e perspectivas. J Manage Prim Heal Care. 2017;8(2):290-308.

11. Lima KMSV, Silva KL, Tesser CD. Integrative and complementary practices and the relationship with health promotion: experience of a municipal healthcare service. Interface (Botucatu) [Internet]. 2013 [acesso em 2017 Jul 17];18(49):261-72. Disponível em: http://www.scielo.br/scielo.php?pid=S141432832014005030133\&script=sci_abstract

12. Contatore AO, Barros NF, Durval MR, Barrio PCCC, Coutinho BD, Santos JA, et al. Uso, cuidado e política das práticas integrativas e complementares na Atenção Primária à Saúde. Ciênc Saúde Colet. 2015;20(10):3263-73.

13. Campos GW, Barros RB, Castro AM. Avaliação de política nacional de promoção da saúde. Ciênc Saúde Colet. 2004;9(3):745-49.

14. Secretaria Municipal da Saúde de Curitiba. Conferências Municipais [Internet]. [data desconhecida] [acesso em 2017 Jul 17]. Disponível em: http://www.saude.curitiba.pr.gov.br/cms/conferencias.html

15. Soares DP, Coelho AM, Silva LEA, Silva RJR, Linard LLP, Fernandes MC. Fatores intervenientes das práticas integrativas e complementares em saúde na Atenção Básica pelos enfermeiros. Rev Enferm Atenção Saúde. 2019;8(1):93-102.

16. Randow R, Campos KC, Roquete FF, Silva LTH, Duarte VES, Guerra VA. Periferização das práticas integrativas e complementares na atenção primária à saúde: desafios da implantação do Lian Gong como prática de promoção à saúde. Rev Bras Promoç Saúde. 2016;29(Supl):111-7. 
17. Nascimento MC, Barros NF, Nogueira MI, Luz MT. A categoria racionalidade médica e uma nova epistemologia em saúde. Ciênc Saúde Colet. 2013;18(12): 3595-3604.

18. Marsiglia RMG. Perfil dos trabalhadores na atenção básica em saúde no município de São Paulo: região norte e central da cidade. Rev Saúde Soc. 2011;20(4):900-11.

19. Thiago SCS, Tesser CD. Percepção de médicos e enfermeiros da estratégia de saúde da família sobre terapias complementares. Rev Saúde Pública. 2011;45(2):249-57.

20. Galhardi WMP, Barros NF, Leite-Mor ACMB. Institucionalização de práticas integrativas e complementares no sistema único de saúde. Hist Cienc Saude-Manguinhos. 2014;21(1):361-3.

21. Plácido AL, Morais KCS, Silva CP, Tavares FM. Percepção dos gestores das Unidades Básicas de Saúde Sobre as Práticas Integrativas e Complementares. Id on Line Rev Mult Psic. 2019;13(43):465-72.

22. Sousa IMC, Bodstein RCA, Tesser CD, Santos FAZ, Hortale VA. Práticas integrativas e complementares: oferta e produção de atendimentos no SUS e em municípios selecionados. Cad Saúde Pública. 2012;28(11):2143-54.

23. Cunha GT. A construção da clínica ampliada na atenção básica. São Paulo: Hucitec; 2005.

24. Gontijo MBA, Nunes MF. Práticas integrativas e complementares: conhecimento e credibilidade de profissionais no serviço público de saúde. Rev Trab Educ Saúd. 2017;15(1):301-20.

25. Fontanella F, Speck FP, Piovezan AP, Kulkamp IC. Conhecimento, acesso e aceitação das práticas integrativas e complementares em saúde por uma comunidade usuária do Sistema Único de Saúde na cidade de Tubarão/SC. ACM Arq Catarin Med. 2007;36(2):69-74.

26. Boing AC, Ribeiro Santiago PH, Tesser CD, Furlan IL, Bertoldi AD, Boing AF. Prevalence and associated factors with integrative and complementary practices use in Brazil. Complement Ther Clin Pract. 2019;27(37):1.

27. Ischkanian PC, Pelicioni MCF. Desafios das práticas integrativas e complementares no SUS visando a promoção da saúde. Rev Bras Crescimento Desenv Hum. 2011;22(1):233-8.

28. National Institutes of Health, Department of Health and Human Services. Complementary and Alternative Medicine: what people aged 50 and older discuss with their health care providers [Internet]. 2011 [acesso em 2017 Jan 20]. Disponível em: https://nccih.nih.gov/sites/nccam.nih.gov/files/news/camstats/2010/NCCAM_ aarp_survey.pdf

29. Galvanese ATC, Barros NF, Oliveira AFPL. Contribuições e desafios das práticas corporais e meditativas à promoção da saúde na rede pública de atenção primária no munícipio de São Paulo, Brasil. Cad Saúde Pública. 2017;33(12):1-13.

30. Carvalho JLS, Nobrega MPSS. Práticas integrativas e complementares como recurso de saúde mental na Atenção Básica. Rev Gaúch Enferm [Internet]. 2017 [acesso em 2017 Jan 20];38(4):e2017-0014. Disponível em: http://www.scielo.br/pdf/rgenf/v38n4/1983-1447-rgenf-38-04-e2017-0014.pdf

\author{
Endereço para correspondência: \\ Luciana Elisabete Savaris \\ Universidade Federal do Paraná - UFPR \\ Programa de Pós-Graduação em Saúde Comunitária \\ Av. Prefeito Lothário Meissner, 632 \\ Bairro: Jardim Botânico \\ CEP: 80210-170 - Curitiba - PR - Brasil \\ E-mail: lucianaesavaris@gmail.com
}

Como citar: Savaris LE, Böger B, Savian AC, Jansen AS, Silva MZ. Práticas integrativas e complementares: análise documental e o olhar de profissionais da saúde. Rev Bras Promoç Saúde. 2019;32:9439. 